Check for updates

Cite this: RSC Adv., 2017, 7, 54475

\title{
Graphene oxide/ $\beta$-cyclodextrin composite as fiber coating for high efficiency headspace solid phase microextraction of organophosphate ester flame retardants in environmental water $\dagger$
}

\begin{abstract}
Jiali Deng, ${ }^{a}$ Pengcheng Zhang, ${ }^{b}$ Tingting Jin, ${ }^{a}$ Hongbin Zhou ${ }^{a}$ and Jing Cheng (D) *a
A novel solid-phase microextraction fiber was prepared using graphene oxide/ $\beta$-cyclodextrin (GO/ $\beta$-CD) composite as a coating material immobilized on a stainless steel wire using sol-gel technique. The coating has large surface area with uniform porous structure, stable performance at high temperature, and good coating preparation reproducibility. The prepared GO/ $\beta-C D$ sol-gel stainless steel fiber used as a headspace solid phase microextraction (HS-SPME) fiber was evaluated by the determination of organophosphate ester flame retardants (OPFRs) in water samples coupled to a gas chromatography/ nitrogen phosphorus detector (GC/NPD). The studied OPFRs have a wide range of polarities and volatilities, and are not easily extracted by conventional commercial SPME fibers simultaneously. The prepared GO/ $\beta-C D$ SPME fiber showed good extraction performance for all the studied OPFRs. The developed HS-SPME-GC/NPD method provided low limits of detection ranging between 1.1 and $60.4 \mathrm{ng} \mathrm{L}^{-1}$. Good intra- and inter-day precision expressed as relative standard deviations for a single fiber were in the range of $2.2-9.6 \%$ and $2.7-9.7 \%$, respectively, and fiber-to-fiber reproducibility was in the range of $6.3-10.9 \%$. The calibration curves were linear in the concentration range from 10 to $50000 \mathrm{ng} \mathrm{L}^{-1}(R>0.9955)$. The developed method was applied for the analysis of tap, lake and river water samples and the relative recoveries were found to be in the range from 82.1 to $116.9 \%$. Based on these features, the proposed fiber has great potential for widespread use as an effective and useful extraction tool.
\end{abstract}

Received 18th July 2017

Accepted 7th November 2017

DOI: $10.1039 / c 7 r a 07903 f$

rsc.li/rsc-advances

\section{Introduction}

For several decades, a variety of flame retardant additives have been applied to polymers and resins found in commercial products, including electronics, furniture, and textiles, to serve to slow the combustion of treated materials to meet flammability standards. Organophosphate flame retardants (OPFRs) are used as replacements for the commercial polybrominated diphenyl ethers (PBDEs) that were phased out in 2004 owing to their persistence, bioaccumulation, and toxicity. ${ }^{1,2}$ Worldwide restrictions on the use of PBDEs in new products have resulted in the increasing use of OPFRs. The annual consumption of OPFRs in the EU increased from 84000 tons in 2004 to 91000 tons in $2006 .{ }^{3}$ OPFRs are just mixed and not chemically bonded with the materials, thus they can easily leach out of the material

${ }^{a}$ Key Laboratory of Pesticide and Chemical Biology, Ministry of Education, Institute of Environmental Chemistry, College of Chemistry, Central China Normal University, Wuhan 430079, China. E-mail: chengjingok@mail.ccnu.edu.cn; Tel: +86-27-67867961 ${ }^{b}$ Department of Biological Science and Technology, School of Environmental Studies, China University of Geosciences, Wuhan 430074, China

$\dagger$ Electronic supplementary information (ESI) available. See DOI: 10.1039/c7ra07903f into the environment via volatilization, abrasion, and dissolution. As a result of these processes, OPFRs are widely distributed in the environment. Although OPFRs are generally considered to be less toxic and harmful than the brominated flame retardants (BFRs), recent studies showed that many kinds of OPFRs have obvious nerve toxicity, carcinogenicity, genetic toxicity, and can cause skin irritation and dermatitis, ${ }^{4-7}$ and especially halogenated OPFRs may affect neurodevelopment with similar or greater potency compared to organophosphorus pesticide and PBDEs organic pollutants. ${ }^{4}$ Furthermore, some of these chemicals (e.g. chlorinated alkyl phosphates) have a low degradation potential and thus may be persistent. ${ }^{8}$ The toxicity and growing usage of OPFRs raised concerns among authorities, and then the analysis of them in environmental media is therefore a hot spot of analytical research at present., ${ }^{9,10}$

In complex environmental media, the analyte is often found at trace level of concentration. As a result, the step of pretreatment is the most challenging and important in the whole analytical procedure. Until now, a number of pretreatment techniques have attempted to extract OPFRs from environmental samples, including liquid-liquid extraction (LLE) ${ }^{11}$ solid-phase extraction (SPE) ${ }^{12}$ membrane-assisted solvent 
extraction (MASE) ${ }^{13}$ dispersive liquid-liquid microextraction (DLLME) ${ }^{14}$ microporous membrane liquid-liquid extraction (MMLLE), ${ }^{15}$ hollow fiber liquid phase microextraction (HFLPME $^{16}$ and solid phase microextraction (SPME). ${ }^{9,10}$ LLE and SPE have been the most often used extraction methods. However, these methods are tedious, labor-intensive and consume large amounts of organic solvents, and suffer from poor recovery of tri (2-chloroethyl) phosphate (TCEP) $(31 \%)^{11}$ or tri(2-ethylhexyl) phosphate (TEHP) (50\%). ${ }^{12}$ MASE employs a nonporous polypropylene membrane to improve the selectivity in the LLE of OPFRs from water samples, but the extraction efficiency in MASE for the most polar TCEP only $5 \%$, and MASE is also quite time-consuming $(3 \mathrm{~h}) .{ }^{13}$ DLLME was introduced since it drastically reduces organic solvents consumption which usually happened in the classical large-scale methods. However, DLLME exhibited unsatisfactory recovery for TEHP (about $40 \%$ ). ${ }^{14}$ Although MMLLE is simple, inexpensive and has no carryover problems, it is unsuitable for the determination of TCEP and TEHP in water samples. ${ }^{15}$ Additionally, the extraction efficiencies of HF-LPME for both TEHP and TCEP were lower than other OPFRs and resulted in lower recoveries as well. ${ }^{16}$

Solid-phase microextraction (SPME) is based on the equilibrium of the target analytes between the sample and fiber coating. Thus, the stationary phase coated onto the fiber plays a key role for the extraction efficiency. ${ }^{17}$ There are some commercially available SPME fibers, such as polydimethylsiloxane (PDMS), polyacrylate (PA), divinylbenzene (DVB), carboxen (CAR), and carbowax (CW), and the homemade coating materials include polypyrrole, ${ }^{18}$ polyaniline ${ }^{19}$ polymeric ionic liquid, ${ }^{20}$ anodized aluminum wire, ${ }^{21}$ ionic liquids, ${ }^{9}$ metal-organic framework, ${ }^{22}$ carbon materials based coatings $^{23-25}$ and so on. The commercial polydimethylsiloxane (PDMS)/divinylbenzene (DVB) SPME fiber and home-made ionic liquid-based sol-gel SPME fiber have been reported to extract OPFRs in environmental media. ${ }^{9,10}$ However, the commercial PDMS/DVB fiber used in headspace-solid phase microextraction (HS-SPME) method, was still not suitable for extracting TEHP due to the low recovery $(26.7 \%)$ and unacceptable deviation $(64.8 \%),{ }^{9}$ and a home-made ionic liquid-based sol-gel SPME fiber has been reported to extract OPFRs from environmental water samples and exhibited good extraction capability and precisions when it applied for the simultaneous extraction of different polarity OPFRs, even including TCEP and TEHP. ${ }^{10}$ However, ionic liquid is expensive and adsorbs water so easily in the air that it is not suitable for practical application.

Graphene oxide (GO), as a graphene precursor, exhibits similar adsorption ability to graphene which shows excellent extraction performance as coating materials of SPME fiber. ${ }^{26}$ Compared with graphene, GO abounds with many oxygencontaining chemical groups across the basal planes, such as epoxy groups, hydroxyl groups, and carboxyl groups, which indicates that it can be readily interacted with other materials. ${ }^{27}$ Furthermore, GO is much cheaper than graphene, suitable for large scale production. Therefore, GO is more practical than graphene as an adsorbent material. However, there are many hydroxy and epoxy groups on the GO sheets which make GO adsorb polar molecules easily through electrostatic interaction.
Thus, introducing a new functional molecule to GO through covalent or noncovalent strategies to bring new or enhanced functions to GO is highly desirable and important.

Cyclodextrins (CDs) are cyclic oligosaccharides consisting of six, seven, or eight $(\alpha-1,4)$-linked-D-glucopyranose units $(\alpha, \beta$, or $\gamma$-CD, respectively), which are toroidal in shape with a hydrophobic inner cavity and a hydrophilic exterior. ${ }^{28,29}$ Such characteristics make them selectively bind many kinds of inorganic, organic, and biological molecules into their cavities, even if only partially, to form supramolecular complexes without structural changes through host-guest interactions (van der Waals force, hydrophobic interaction, electrostatic affinity, dipole-dipole interaction, and hydrogen bonding). ${ }^{29} \beta$-CD is the most largely produced CD used in many fields, including pharmaceuticals, food, cosmetics, chemical products, and technologies. ${ }^{30}$ The applications include chemical separations, ${ }^{31,32}$ adsorbents, ${ }^{27,33}$ food processing, ${ }^{34}$ and pharmaceutical excipients. ${ }^{35}$ Once GO is functionalized with $\beta-\mathrm{CD}$, it is likely to gain a new material simultaneously having the properties of both GO (high surfaceto-weight ratio and large delocalized-electron system) and $\beta$-CD (high supramolecular recognition and enrichment capability) through combining their individual characteristics. However, as far as we know, there has been no report yet for the application of the $\beta$-CD-functionalized GO as a fiber coating material in SPME for the analysis of volatile or semivolatile OPFRs.

The new preparation methods of a SPME fiber, including direct use of uncoated fibers, ${ }^{36}$ epoxy-glue solid sorbent, ${ }^{37}$ physical deposition, ${ }^{38}$ electrochemical deposition, ${ }^{39}$ and sol-gel technique. ${ }^{9,18}$ Among the various coating preparation methods, sol-gel technique is popular to improve the performance of SPME coating. The synthetic conditions of sol-gel are usually mild, and the coating prepared by the sol-gel technique is often of high purity and homogeneity, high thermal and solvent stability, and high surface areas with porous structure. ${ }^{40}$ To overcome the fragile of fused silica fibers, the coating fiber was immobilized on stainless steel wire due to its rigidity, low cost, non-toxicity and durability. The integrated use of the sol-gel technique and the stainless steel wire for the preparation of the SPME fibers would take the advantages of the both and possibly enhance the durability of the prepared fiber.

In this work, $\beta$-CD decorated GO material was prepared under the conditions of ultrasound irradiation. The $\beta$-CD macromolecules were covalently grafted onto the surface of GO through 3-aminopropyltriethoxysilane for forming amido bond with GO and forming silicon oxygen bond with $\beta$-CD. A novel $\beta$ CD-GO-coated SPME fiber was prepared by immobilizing the $\beta$ CD-GO through a sol-gel technique onto a prefunctionalized stainless-steel wire. The extraction performance of the $\beta$-CDGO-coated fiber was evaluated with volatile and semivolatile OPFRs as the model analytes because of their toxicity and widespread environmental occurrence. The main experimental parameters including the extraction time and temperature, desorption temperature and time, the ratio of the volume of water solution to headspace (HS), stirring rate, and ionic strength were investigated to achieve a good extraction efficiency. In addition, the fiber lifetime was also evaluated. Finally, a HS-SPME with the $\beta$-CD-GO coated fiber followed by GC with 
nitrogen phosphorus detector (NPD) for the determination of some OPFRs was developed. The method was applied for the determination of the OPFRs in tap, lake and river water samples.

\section{Experimental}

\subsection{Reagents and materials}

Graphite powder (50 meshes), potassium permanganate $\left(\mathrm{KMnO}_{4}\right)$, sulfuric acid $\left(\mathrm{H}_{2} \mathrm{SO}_{4}, 98 \%\right)$, hydrogen peroxide $\left(\mathrm{H}_{2} \mathrm{O}_{2}\right)$, sodium chloride $(\mathrm{NaCl}), \beta$-cyclodextrin ( $\beta$ - $\mathrm{CD})$, ethanol, acetone, concentrated hydrochloric acid and other chemicals were all of analytical grade and purchased from Sinopharm Chemical Reagent Co., Ltd. (Shanghai, China). Methyltrimethoxysilane (MTMOS, 97\%), poly (methylhydrosiloxane) (PMHS), and (3mercaptopropyl) trimethoxysilane (MPTS, 95\%) were purchased from J\&K Scientific Ltd. (Beijing, China). Trifluoroacetic acid (TFA) and 3-aminopropyltriethoxysilane (3-APTES) were purchased from Aladdin Reagent Co., Ltd. (Shanghai, China). Tripropyl phosphate (TPrP), tri- $n$-butyl phosphate (TnBP), tri(2chloroethyl) phosphate (TCEP) and tri(2-ethylhexyl) phosphate (TEHP) were purchased from Dr Ehrenstorfer Gmbh (Augsburg, Germany). Tri(2-chloroisopropyl) phosphate (TCPP), 2-ethylhexyl diphenyl phosphate (EHDPP) were obtained from SigmaAldrich (Louis, USA), and triphenyl phosphate (TPhP), tricresyl phosphate (TCrP) were purchased from Aucc stand (NewHaven, USA). The physicochemical properties of the nine OPFRs are listed in Table S1 (see ESI†े).

\subsection{Apparatus}

A magnetic stirrer (model: MC-MAG HS7) purchased from IKA instrument equipment Co., Ltd. (IKA, Guangzhou, China) was employed for stirring and heating samples during the extraction. Ultrasonic instrument KQ-100DE was purchased from Kunshan Ultrasonic Instrument Co., Ltd. (Jiangsu, China). The commercial fibers coated with $100 \mu \mathrm{m}$ thickness of polydimethylsiloxane (PDMS) coating and $80 \mu \mathrm{m}$ thickness of polyamide (PA) coating were used for comparative purposes.

The analytes were separated and detected on a TRACE 1300 system (Thermo Fisher Scientific, MA, USA), equipped with split/splitless injector, a TR-1 capillary column $(30 \mathrm{~m} \times$ $0.32 \mathrm{~mm}$ i.d., $0.25 \mathrm{~m}$ film thickness) and nitrogen-phosphorous detector (NPD). Ultrapure nitrogen $(>99.999 \%)$ was used as carrier gas at a constant flow of $2 \mathrm{~mL} \mathrm{~min}{ }^{-1}$ and it also used as

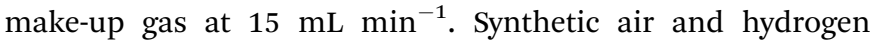
$(99.999 \%)$ were used as detector gases at flow rates of 60 and 2.3 $\mathrm{mL} \min ^{-1}$, respectively. The oven temperature was programmed as follows: initial temperature from $80^{\circ} \mathrm{C}$ (held for 1.0 min), heating at $20^{\circ} \mathrm{C} \mathrm{min}^{-1}$ to $170^{\circ} \mathrm{C}$ (held for $1.0 \mathrm{~min}$ ), then at $4{ }^{\circ} \mathrm{C} \min ^{-1}$ to $270{ }^{\circ} \mathrm{C}$ (held for $1.0 \mathrm{~min}$ ). The injector and the detector temperatures were set at 270 and $320{ }^{\circ} \mathrm{C}$, respectively.

Surface structure of the prepared fiber was investigated by JSM-6700F scanning electron microscopy (SEM) (FESEM, JEOL, Japan). Fourier transform infrared spectroscopy was performed with a Thermo Nicolet Is50 FT-IR instrument (Boston, MA, USA). The thermal stability of the developed coatings was investigated by a STA 409 PC thermogravimetric analysis (TGA) and differential scanning calorimetry (DSC) instrument. The samples were heated from $30{ }^{\circ} \mathrm{C}$ to $500{ }^{\circ} \mathrm{C}$ under a nitrogen atmosphere at a heating rate of $10^{\circ} \mathrm{C} \mathrm{min}^{-1}$.

\subsection{Water sample}

River water was collected from the Changjiang River (Wuhan, China). Lake water was collected from East Lake (Wuhan, China). Tap water was collected from Central China Normal University (Wuhan, China). All water samples were stored in amber glass bottles at $4{ }^{\circ} \mathrm{C}$ in darkness before use.

\subsection{Preparation of GO/ $\beta$-CD sol-gel stainless steel fiber and HS-SPME procedures}

As shown in Fig. 1 , the whole process of preparation of $\mathrm{GO} / \beta-\mathrm{CD}$ sol-gel stainless steel fiber and its HS-SPME procedures were presented as follows:

Firstly, the stainless steel wire was modified. As shown in Fig. S1 (see ESI $\dagger$ ), a stainless steel wire (about $2.5 \mathrm{~cm}$ of the wire end) vibrated ultrasonically in ethanol, acetone and again in ethanol for $10 \mathrm{~min}$ in each to remove any contaminants. The MPTS was used to modify the surface of the steel wire. For modification, $1.5 \mathrm{~cm}$ of the wire end was dipping into a solution of $10^{-3} \mathrm{~mol} \mathrm{~L}^{-1}$ MPTS in ethanol for $3 \mathrm{~h}$. It is generally known that under ambient conditions, surfaces of common metals and alloys such as stainless steel are covered with oxide species. They can react with MPTS to form a monolayer..$^{39}$ Afterwards, the wires were rinsed with ethanol and dried under nitrogen atmosphere. Finally, the modified stainless steel wire was immersed in hydrochloric acid ( $0.1 \mathrm{M}$ in water) for $1 \mathrm{~h}$ to hydrolyze the methoxy groups.

Secondly, GO/ $\beta$-CD composite was prepared. Graphene oxide (GO) was prepared by oxidizing natural graphite powders and then transformed into GO sheets. ${ }^{39}$ Then, the schematic synthesis process for GO/ $\beta$-CD composite was shown in Fig. S2. $\dagger$ $30 \mathrm{mg}$ GO was dissolved in $30 \mathrm{~mL}$ DMF by sonication and then $3 \mathrm{~mL}$ of APTES was added to the above GO/DMF mixture. The mixture was stirred at $70{ }^{\circ} \mathrm{C}$ for $30 \mathrm{~min}$. GO would react with APTES by forming amido bond between the - $\mathrm{COOH}$, epoxy groups from $\mathrm{GO}$ and $-\mathrm{NH}_{2}$ from APTES. After the reaction finished, $1 \mathrm{~g} \beta$-CD was added to the obtained mixture, after stirring $5 \mathrm{~h}$, the mixture was centrifuged at $10000 \mathrm{rpm}$ for $8 \mathrm{~min}$ and then, the resultant precipitate was washed by DMF to remove the unreacted $\beta-\mathrm{CD}$ and finally dried under at $60{ }^{\circ} \mathrm{C}$ to yield $\mathrm{GO} / \beta-\mathrm{CD}$ composite.

Thirdly, $3 \mathrm{mg}$ of GO/ $\beta$-CD composite was dissolved in $100 \mu \mathrm{L}$ DMF in a $1.5 \mathrm{~mL}$ micro-centrifuge tube, and the tube was vibrated sonically for $2.5 \mathrm{~h}$. Then $300 \mu \mathrm{L}$ MTMOS and $50 \mu \mathrm{L}$ PMHS, used as sol-gel precursor and deactivation reagent, respectively, were added to the above-mentioned microcentrifuge tube and vibrated sonically for $20 \mathrm{~min}$. $60 \mu \mathrm{L} 95 \%$ TFA solution, used as acid catalyst, was added and mixed for $10 \mathrm{~min}$. The GO/ $\beta$-CD composite sol-gel solution was obtained. Finally, the modified stainless fire wire was vertically dipped into the GO/ $\beta$-CD sol-gel solution $1.5 \mathrm{~cm}$ deep for $10 \mathrm{~min}$ and a gel coating was formed on the wire surface. Then, it was pulled 

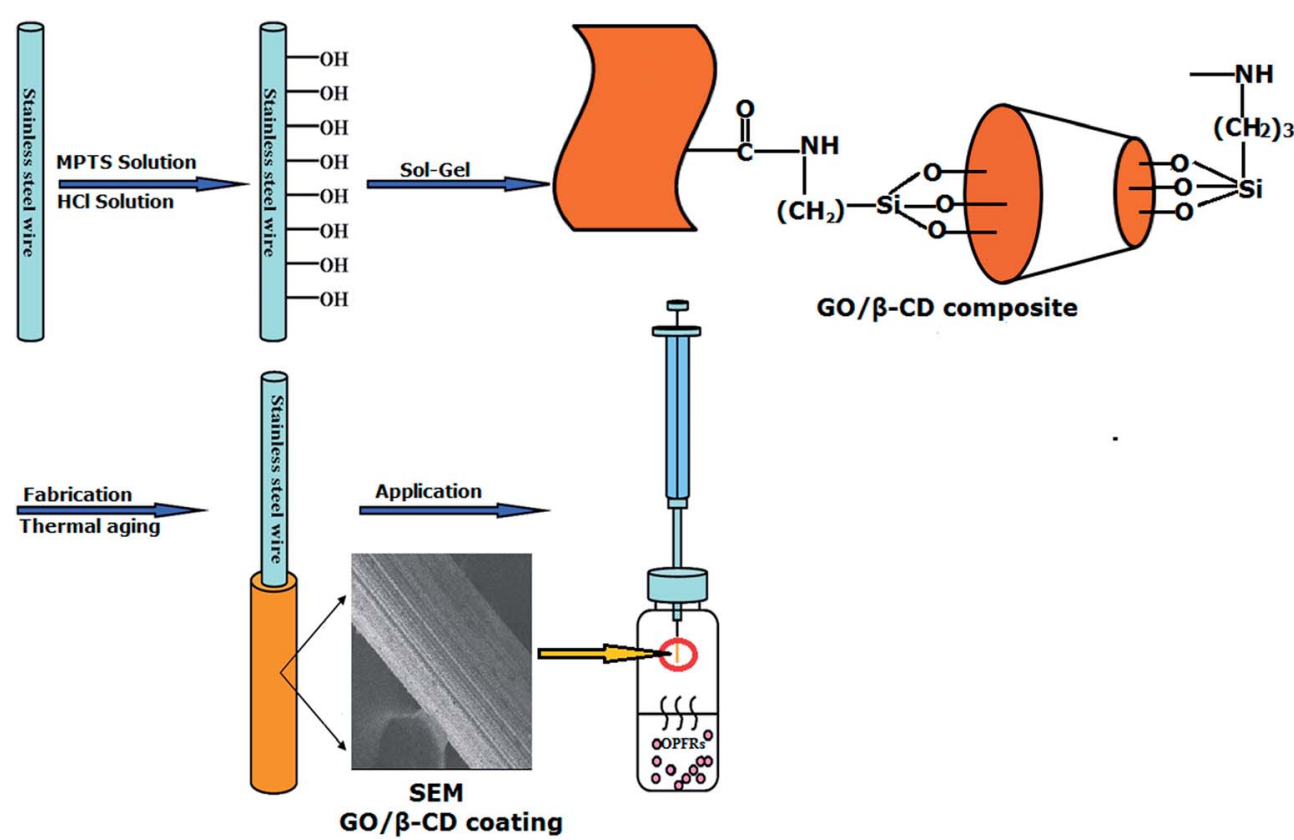

Fig. 1 Schematic presentation of preparation of GO/ $\beta-C D$ sol-gel stainless steel fiber coating and its application for HS-SPME of OPFRs.

out and dried at room temperature for $20 \mathrm{~min}$. This coating process was repeated several times until the desired thickness of the coating (about $10 \mu \mathrm{m}$ ) was obtained. Then, the coated fiber was put in a drier for curing at room temperature for $24 \mathrm{~h}$. Prior to use, the fiber was assembled into a $5 \mu \mathrm{L}$ GC microsyringe and then conditioned in the GC injector at $100{ }^{\circ} \mathrm{C}$ for $0.5 \mathrm{~h}$, each additional $50{ }^{\circ} \mathrm{C}$ for $0.5 \mathrm{~h}$ to $270{ }^{\circ} \mathrm{C}$.

The sol-gel coated fiber was prepared with the same procedures in the absence of GO/ $\beta$-CD composite. The GO sol-gel coating fiber and $\beta$-CD sol-gel coating fiber were prepared with the same procedures as the GO/ $\beta$-CD coating fiber except that GO and $\beta-C D$ were used instead of $\mathrm{GO} / \beta-\mathrm{CD}$ composite, respectively.

Finally, HS-SPME procedures were performed in a $20 \mathrm{~mL}$ amber glass vial containing $14 \mathrm{~mL}$ aqueous solution which was tightly sealed with an aluminum cap. After adding $4.2 \mathrm{~g} \mathrm{NaCl}$, the $20 \mathrm{~mL}$ amber glass vial with the fiber exposed to the headspace above the sample was put in a $80{ }^{\circ} \mathrm{C}$ oil bath on the magnetic stirrer for $30 \mathrm{~min}$ at a constant stirring rate of $600 \mathrm{rpm}$. After extraction, the fiber was withdrawn into the needle of the GC micro-syringe and removed from the sample vial. Finally, the GC micro-syringe was inserted into the GC injector to desorb the analytes at $270{ }^{\circ} \mathrm{C}$ for $4 \mathrm{~min}$ and then for GC/NPD analysis.

\section{Results and discussion}

\subsection{Characterization of the prepared fiber}

The SEM images of a stainless steel fiber, the prepared $\beta$-CD sol-gel coating bonded on the stainless steel fiber, the prepared GO sol-gel coating bonded on the stainless steel fiber and the prepared GO/ $\beta$-CD sol-gel coating bonded on the stainless steel fiber were illustrated in Fig. S3a-d, $\uparrow$ respectively. Fig. S3 $\uparrow$ let us see clearly the morphological structure of $\beta$-CD sol-gel coating, GO sol-gel coating and GO/ $\beta$-CD coating which were homogeneously bonded on the stainless steel fiber with fluted structures. GO sol-gel coating owns similar fluted morphological structure with $\mathrm{GO} / \beta-\mathrm{CD}$ coating. Both GO sol-gel coating and $\mathrm{GO} / \beta$-CD coating are much rougher than $\beta$-CD coating.

As shown in Fig. 2a, the strong adsorption peak around $3450 \mathrm{~cm}^{-1}$ in the FT-IR spectra was the $\mathrm{O}-\mathrm{H}$ stretching vibration from both GO and $\beta-\mathrm{CD}$. The $\mathrm{O}-\mathrm{H}$ stretching vibration adsorption peaks of both $\mathrm{GO} / \beta-\mathrm{CD}$ composite and $\mathrm{GO} / \beta-\mathrm{CD}$ solgel coating moved to lower wave number at around $3420 \mathrm{~cm}^{-1}$ due to the formation of hydrogen bond between GO and $\beta$-CD. The $\mathrm{C}=\mathrm{O}$ stretching vibration adsorption peak at $1719 \mathrm{~cm}^{-1}$ was moved to lower wave number at $1680 \mathrm{~cm}^{-1}$ and $1670 \mathrm{~cm}^{-1}$ due to the formation of amido bond from the GO/ $\beta-\mathrm{CD}$ composite and GO/ $\beta$-CD sol-gel coating, respectively. The typical $\mathrm{C}-\mathrm{O} / \mathrm{C}-\mathrm{C}$ stretching vibrations at $1030 / 1080 \mathrm{~cm}^{-1}$ and $\mathrm{CH}_{2}$ stretching vibrations at $2928 / 2988 \mathrm{~cm}^{-1}$ of $\beta-\mathrm{CD}$ were found in $\mathrm{GO} / \beta-\mathrm{CD}$ composite and $\mathrm{GO} / \beta-\mathrm{CD}$ sol-gel coating. All these results confirmed that the $\beta$-CD were covalently bonded to the surface of GO. As illustrated in Fig. 2b, the thermal stability of GO/ $\beta$-CD sol-gel coating was evaluated by the TGA and DSC. There is no obvious weight loss (only $5 \%$ weight loss) from $70-$ $350{ }^{\circ} \mathrm{C}$ which might be due to the loss of water. The result indicates that the coating has good thermal stability and it can endure the high temperature in the GC/NPD injector. The lifetime of the fiber is very crucial for practical application. The high temperature of the injection port of GC and the solvent in the matrix are the main factors which damaged the coating. As shown in Fig. $\mathrm{S} 4, \dagger$ the extraction performance of the prepared fiber without significant deterioration after being used more than 150 times in an adsorption/desorption cycle. The long 

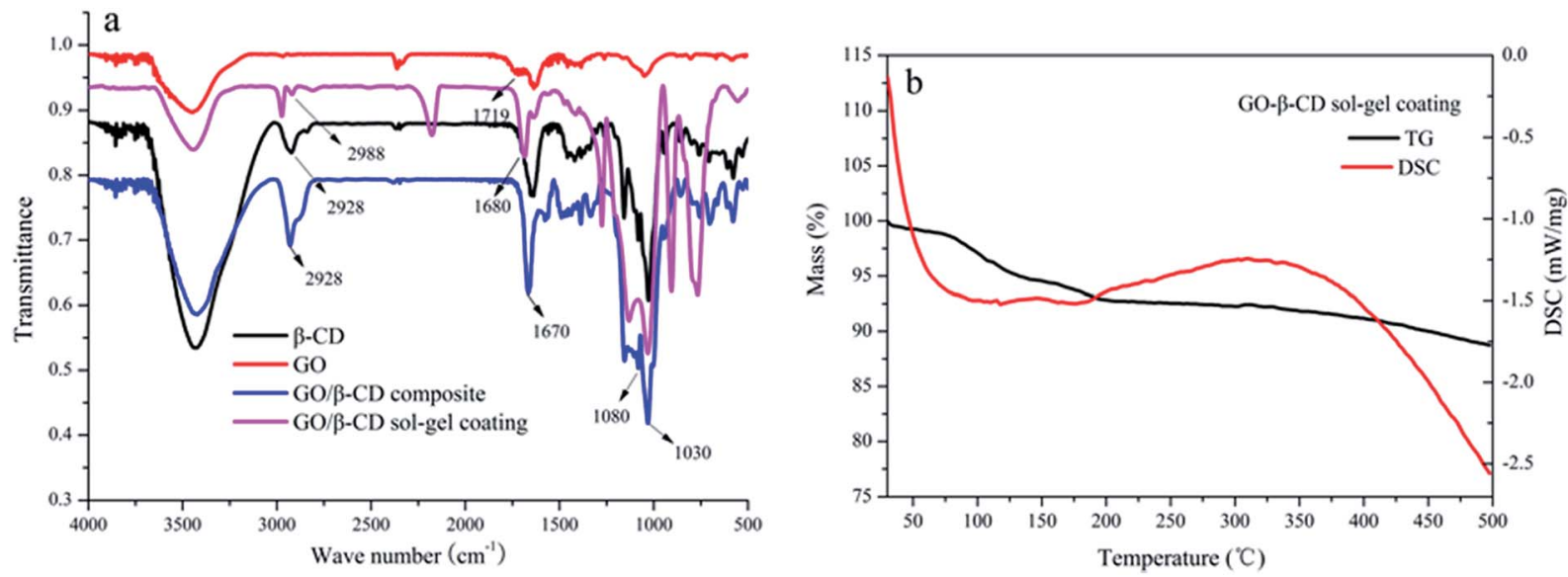

Fig. 2 FT-IR spectra of $\beta-C D, G O, G O / \beta-C D$ composite and GO/ $\beta-C D$ sol-gel coating (a). TGA and DSC curves of the GO/ $\beta-C D$ sol-gel coating (b).

lifetime of the prepared fiber may be due to its good thermal and mechanic stability.

As shown in Fig. 3, the $\mathrm{N}_{2}$ adsorption desorption isotherms of sol-gel coating (a), GO sol-gel coating (b), $\beta$-CD sol-gel coating (c) and $\mathrm{GO} / \beta$-CD sol-gel coating (d) all showed IV type curves, which correspond to the capillary condensation phenomenon of porous adsorbents. The hysteresis loops of Fig. 3a-c display H3 type curves, which meant the pore structure was very irregular. While the hysteresis loops of Fig. $3 \mathrm{~d}$ showed a prominent adsorption platform which reflected uniform pore size distribution of GO/ $\beta$-CD sol-gel coating. The pore structure properties are shown in Table 1. The specific BET surface area, mean pore diameter and the mesopore volume of $\mathrm{GO} / \beta$-CD solgel coating were bigger than pure sol-gel coating, GO sol-gel coating and $\beta$-CD sol-gel coating, indicating that the extraction capacity of GO/ $\beta$-CD sol-gel coating fiber would be the highest.

\subsection{Optimization of the HS-SPME conditions}

HS-SPME mode can minimize the matrix interference from sample and prolong the fiber lifetime when it was used for the extraction of the semi-volatile and volatile compounds. ${ }^{40}$ To achieve the best extraction efficiency for the nine OPFRs, the following parameters were investigated and optimized: (a) extraction temperature; (b) extraction time; (c) volume of water sample; (d) stirring rate (e) ionic strength; (f) desorption time. Each experiment was performed in triplicate. Respective data and figures are given in Fig. S5. $\uparrow$ The following experimental conditions were found to give best results: (a) $80^{\circ} \mathrm{C}$ was chosen as the extraction temperature. (b) $30 \mathrm{~min}$ was selected as the preferred extraction time. (c) $14 \mathrm{~mL}$ water was used to perform in $20 \mathrm{~mL}$ sample vial. (d) Stirring rate of $600 \mathrm{rpm}$ was used. (e) $30 \%(\mathrm{w} / \mathrm{v})$ $\mathrm{NaCl}$ was chosen as the optimum content. (f) All desorption processes were performed at $270{ }^{\circ} \mathrm{C}$ for 4 min (Table 2).

\subsection{Method validation}

Under the optimized conditions, the prepared $\mathrm{GO} / \beta-\mathrm{CD}$ sol-gel coating fiber was used for determination of nine OPFRs in different environmental water coupled to GC/NPD. A series of experiments with regard to the linearity, limits of detection (LOD), limits of quantification (LOQs), repeatability and enrichment factors was performed to validate the HS-SPME-GC/ NPD method. As shown in Table 3, the method using a GO/ $\beta-C D$ sol-gel coating stainless steel wire as the HS-SPME fiber showed a wide linear range with correlation coefficients $(R)$ ranging from 0.9955 to 0.9998 . The LODs and LOQs for the nine analytes ranged from 1.1 to $60.4 \mathrm{ng} \mathrm{L}^{-1}$ and 2.7 to $170.5 \mathrm{ng} \mathrm{L}^{-1}$ based on the ratio of signal-to-noise identical to 3 and 10, respectively. The intra- and inter-day precisions of the method, expressed as relative standard deviations (RSDs) were less than 9.7\%. Enrichment factor (EF) was defined as the ratio of the sensitivity of an analyte after extraction to that before extraction (i.e., by direct injection of $1 \mu \mathrm{L}$ of standard solution) using the chromatographic peak area for quantification, EFs for all analytes were in the range from 22.5 to 1307.5 . The higher polarity and boiling point of the TCEP may result in the lower EF than the other OPFRs. The proposed method had high enrichment factors for most analytes because the fiber combined the advantages of $\beta-\mathrm{CD}$ with the hydrophobic interior cavity and hydrophilic peripheral face and GO with large surface and highly delocalized conjugate system of the $\pi$-electron and the $\pi-\pi$ interaction. The fiber-to-fiber reproducibility was also determined using three different $\mathrm{GO} / \beta-\mathrm{CD}$ coating stainless steel fibers fabricated in the same batch, and the RSDs ranged from 6.3 to $10.9 \%$, which suggests that the fabrication of fiber is reproducible.

\subsection{Comparison with the commercial fibers and some published methods}

The extraction efficiency of GO/ $\beta$-CD sol-gel coating fiber was compared with pure sol-gel coating fiber $(10 \mu \mathrm{m})$, GO sol-gel coating $(10 \mu \mathrm{m}), \beta$-CD sol-gel coating fiber $(10 \mu \mathrm{m})$, commercial PDMS $(100 \mu \mathrm{m})$ and PA fiber $(80 \mu \mathrm{m})$ under their optimized conditions, respectively. As shown in Fig. 4, except for the OPFRs containing benzene ring, $\mathrm{GO} / \beta$-CD sol-gel coating fiber showed the highest extraction efficiency among all the other 

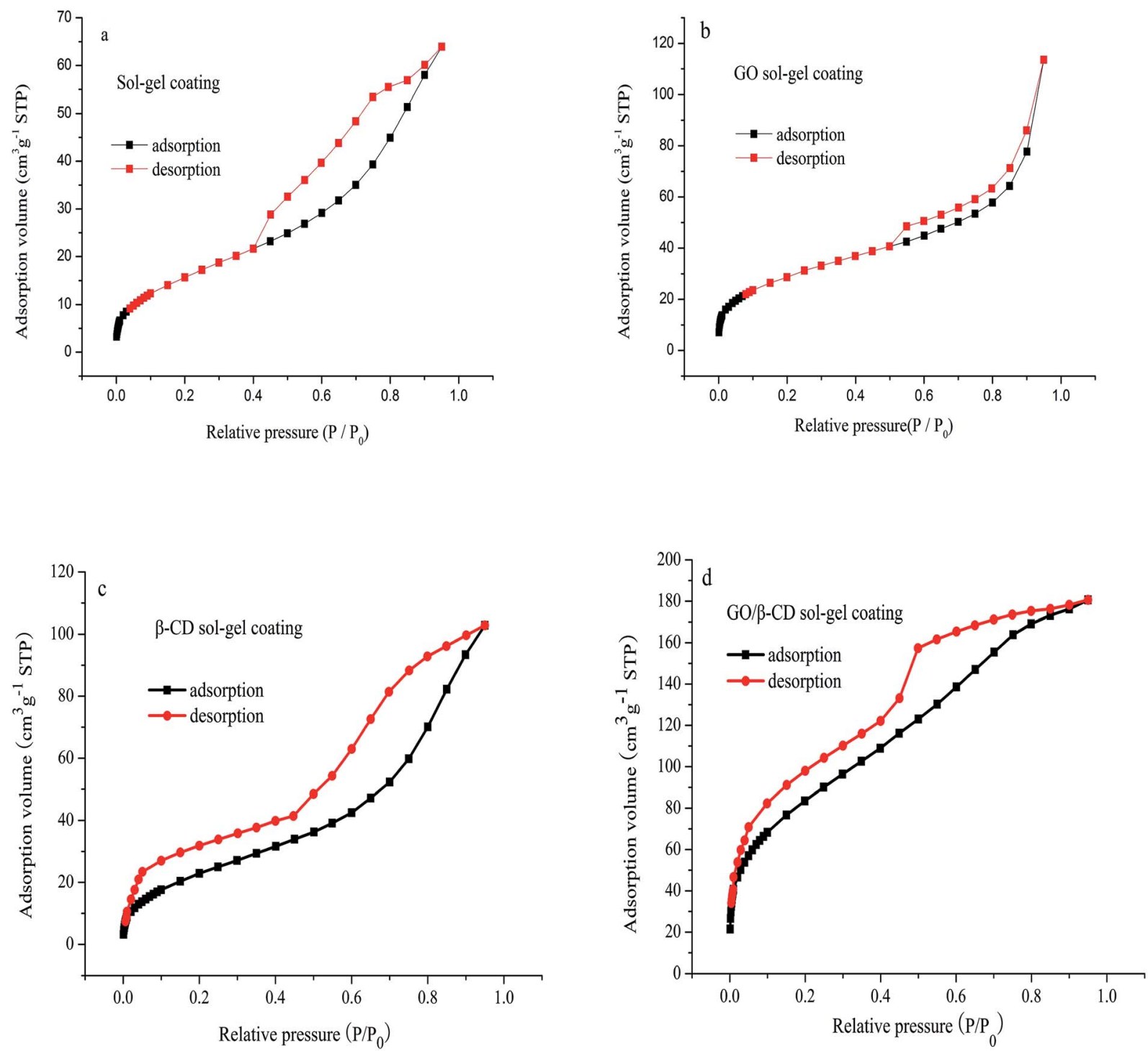

Fig. 3 Nitrogen adsorption-desorption isotherms of the pure sol-gel coating (a); GO sol-gel coating (b); $\beta$-CD sol-gel coating (c) and GO/ $\beta$ CD sol-gel coating (d).

fibers. GO sol-gel coating fiber displayed slightly higher extraction efficiency for three OPFRs containing benzene ring than GO/ $\beta$-CD sol-gel coating fiber. That may be because the GO has higher delocalized conjugate system of the $\pi$-electron and $\pi-\pi$ interaction than $\mathrm{GO} / \beta-\mathrm{CD}$, so it has higher affinity for aromatic compounds. The performance of GO/ $\beta$-CD-coating materials for extraction in molecules with different benzene or non-polar groups will be further deeply investigated in our group.

To illustrate the advantages of the GO/ $\beta$-CD-coating materials as a novel SPME coating fiber, the comparative study of our developed method with other reported sample

Table 1 Pore structure properties of sol-gel coating, GO sol-gel coating, $\beta-C D$-sol-gel coating and GO/ $\beta-C D$ sol-gel coating ${ }^{a}$

\begin{tabular}{|c|c|c|c|}
\hline Samples & Mesopore volume $\left(\mathrm{cm}^{3} \mathrm{~g}^{-1}\right)$ & Mean pore diameter $(\AA)$ & $\operatorname{SBET}\left(\mathrm{m}^{2} \mathrm{~g}^{-1}\right)$ \\
\hline Sol-gel coating & 0.165 & 3.179 & 62.127 \\
\hline GO-sol-gel coating & 0.095 & 7.032 & 107.478 \\
\hline$\beta$-CD/GO-sol-gel coating & 0.280 & 8.079 & 229.375 \\
\hline
\end{tabular}

${ }^{a}$ SBET: specific area calculated according to BET method. 
Table 2 Analytical performances of the GO/ $\beta-C D$ sol-gel coating SPME fiber

\begin{tabular}{|c|c|c|c|c|c|c|c|c|}
\hline \multirow[b]{3}{*}{ Analytes } & \multirow{3}{*}{$\begin{array}{l}\text { Linear range } \\
\left(\mathrm{ng} \mathrm{L}^{-1}\right)\end{array}$} & \multirow[b]{3}{*}{$R$} & \multirow{3}{*}{$\begin{array}{l}\text { LOD } \\
\left(\mathrm{ng} \mathrm{L}^{-1}\right)\end{array}$} & \multirow{3}{*}{$\begin{array}{l}\text { LOQ } \\
\left(\mathrm{ng} \mathrm{L}^{-1}\right)\end{array}$} & \multicolumn{3}{|c|}{$\operatorname{RSD}(\%, n=6)^{a}$} & \multirow[b]{3}{*}{ EFs } \\
\hline & & & & & \multicolumn{2}{|c|}{$\begin{array}{l}\text { Repeatability } \\
\text { (single fiber) }\end{array}$} & \multirow{2}{*}{$\begin{array}{l}\text { Reproducibility } \\
\text { (fiber-to-fiber) }\end{array}$} & \\
\hline & & & & & Intra-day & Inter-day & & \\
\hline TPrP & $50-5 \times 10^{4}$ & 0.9995 & 5.7 & 14.2 & 2.2 & 6.0 & 8.5 & 176.8 \\
\hline TCPP & $100-5 \times 10^{4}$ & 0.9955 & 20.7 & 56.9 & 8.2 & 8.9 & 8.3 & 141.5 \\
\hline TDCP & $100-5 \times 10^{4}$ & 0.9998 & 12.5 & 28.8 & 6.5 & 7.2 & 6.3 & 158.5 \\
\hline TPhP & $50-5 \times 10^{4}$ & 0.9967 & 5.2 & 16.8 & 8.3 & 9.7 & 10.9 & 1307.6 \\
\hline EHDPP & $50-5 \times 10^{4}$ & 0.9970 & 6.9 & 20.4 & 7.2 & 8.1 & 8.4 & 1079.4 \\
\hline TEHP & $50-5 \times 10^{4}$ & 0.9974 & 7.2 & 19.9 & 8.4 & 9.4 & 7.1 & 432.9 \\
\hline TCrP & $50-5 \times 10^{4}$ & 0.9986 & 17.7 & 48.5 & 6.8 & 3.3 & 8.1 & 1270.5 \\
\hline
\end{tabular}

${ }^{a}$ Spiking level: $10 \mu \mathrm{g} \mathrm{L} \mathrm{L}^{-1}$ TnBP, TPrP, TPhP, EHDPP, TCrP, TEHP and $50 \mu \mathrm{g} \mathrm{\textrm {L } ^ { - 1 }}$ TCEP, TCPP, TDCP.

preparation procedures $^{9-16}$ is performed and the results are presented in Table 3. As shown in Table 3, the LODs obtained by the developed $\beta / C D-G O-b a s e d$ sol-gel fiber SPME/GC-NPD method are comparable to those reported in other papers, and the recoveries are better than those published methods.

\subsection{Application for analysis of environmental water}

The feasibility of the proposed HS-SPME/GC method for determining OPFRs in lake water, river water, and tap water was investigated by analyzing $14 \mathrm{~mL}$ of the real water samples spiked with $10 \mu \mathrm{L}$ of acetone. In Fig. 5 and Table 4, no target analytes were detected in the tap water. TnBP $\left(97.7 \mathrm{ng} \mathrm{L}^{-1}\right)$, TCPP (150.4 ng L $\left.{ }^{-1}\right)$, TPhP (162.1 ng L $\left.{ }^{-1}\right)$, TEHP (185.3 $\mathrm{ng} \mathrm{L}^{-1}$ ) detected in the river water, TEHP $\left(205.4 \mathrm{ng} \mathrm{L}^{-1}\right)$ was detected in the lake water. TEHP was detected in both river and lake water due to its poor degradability, which was consistent with Letcher's study. ${ }^{35}$

Recovery testing was carried out by spiking a standard mixture prepared in acetone to $14 \mathrm{~mL}$ of real water matrices and the amount of acetone was kept at $1 \%(\mathrm{v} / \mathrm{v})$. The relative recoveries and the RSDs are listed in Table 4 . The results showed that the relative recoveries of OPFRs $(n=3)$ ranged from 82.1 to $116.9 \%$, and the RSDs were between $3.0 \%$ and $10.4 \%$.

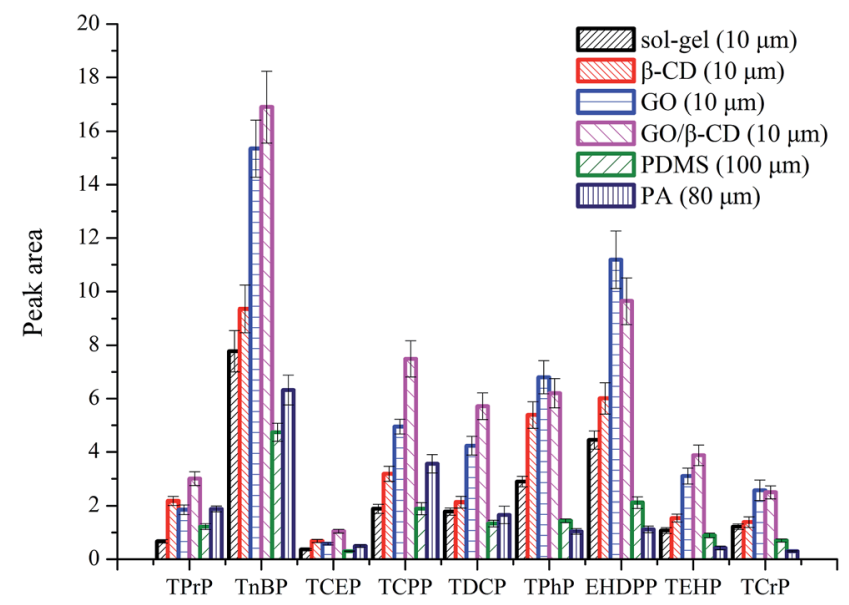

Fig. 4 Comparison of the extraction efficiencies of OPFRs by the bare sol-gel fiber, $\beta$-CD fiber, GO fiber, GO/ $\beta$-CD fiber, PDMS and PA fiber. Experimental conditions: concentration of OPFRs, $50 \mathrm{ng} \mathrm{mL}^{-1} \mathrm{TPrP}$, TnBP, TPhP, EHDPP, TEHP, TCrP, 250 ng $\mathrm{mL}^{-1}$ TCEP, TCPP, TDCP; extraction time, $30 \mathrm{~min}$; extraction temperature, $80^{\circ} \mathrm{C}$; stirring rate, $600 \mathrm{rpm}$; the concentration of salt, 20\% (w/v); desorption time, $4 \mathrm{~min}$.

The results also prove that the proposed method is robust and can be applied to the determination of OPFRs in different water samples with satisfying accuracy and precision. In addition, the

Table 3 Figures of merit of comparable methods for determination of OPFRs

\begin{tabular}{|c|c|c|c|c|c|}
\hline Method & RSD (\%) & $\operatorname{LOD}\left(\mathrm{ng} \mathrm{L}^{-1}\right)$ & Recovery (\%) & EFs & Ref. \\
\hline LLE/GC-MS & $12-33$ & $1.9-6.1$ & $31-107$ & - & 11 \\
\hline SPE/LC-MS & 4-13 & $0.9-24.5$ & $50-128$ & - & 12 \\
\hline MASE/LC-MS/MS & $2-19$ & $3-25$ & $5-98$ & $9-196$ & 13 \\
\hline DLLME/GC-NPD & $2-17$ & $10-80$ & 23-109 & 195-906 & 14 \\
\hline MMLLE/GC-NPD & $4-16$ & $20-120$ & $4-51$ & $34-1400$ & 15 \\
\hline HF-LPME/GC-MS & $2.1-10.4$ & $2.6-120.0$ & $24-132$ & $132-294$ & 16 \\
\hline SPME (PDMS-DVB fiber)/GC-NPD & $5.3-64.8$ & $4.5-7.6$ & $26.7-119.2$ & - & 9 \\
\hline SPME (IL-based sol-gel fiber)/GC-FPD & $3.3-7.6$ & $0.7-11.6$ & $73.2-101.8$ & $13.4-69.1$ & 10 \\
\hline SPME ( $\beta$-CD-GO-based sol-gel fiber $) / G C-$ & $2.2-9.6$ & $1.1-60.4$ & 82.1-116.9 & $22.5-1307.6$ & This study \\
\hline
\end{tabular}
NPD 


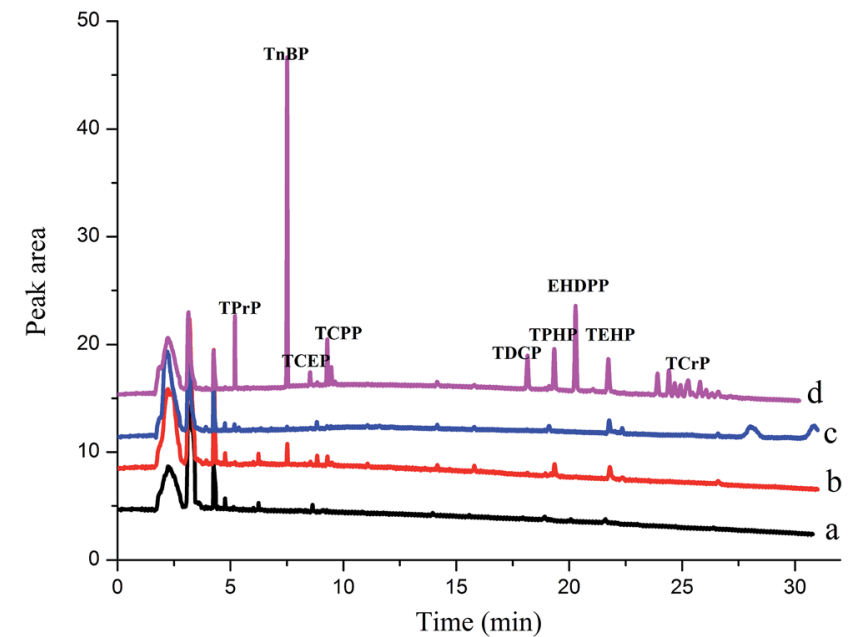

Fig. 5 Chromatograms of blank tap water after extraction (a), blank river water after extraction (b), blank lake water after extraction (c) and river water spiked with OPFRs after extraction (d) (spiking level: $1 \mathrm{ng}$

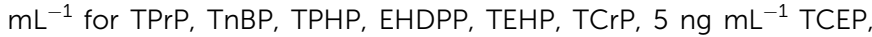
TCPP, TDCP).

Table 4 Recoveries and precisions (RSD, $n=5$ ) of the nine OPFRs in different water obtained by the presented method

\begin{tabular}{|c|c|c|c|c|}
\hline \multirow[b]{2}{*}{ Analytes } & \multirow{2}{*}{$\begin{array}{l}\text { Spiked } \\
\left(\text { ng L L }^{-1}\right)\end{array}$} & \multicolumn{3}{|c|}{ Recovery $\pm \operatorname{RSD}(\%)(n=5)$} \\
\hline & & Lake water & River water & Tap water \\
\hline \multirow[t]{3}{*}{ TPrP } & 0 & nd & nd & nd \\
\hline & 100 & $97.0 \pm 3.0$ & $97.0 \pm 3.0$ & $96.7 \pm 6.7$ \\
\hline & 1000 & $82.1 \pm 4.3$ & $83.0 \pm 4.3$ & $87.0 \pm 7.7$ \\
\hline \multirow[t]{3}{*}{ TnBP } & 0 & nd & $97.7 \pm 7.8$ & nd \\
\hline & 100 & $96.0 \pm 5.2$ & $83.5 \pm 5.2$ & $97.2 \pm 3.9$ \\
\hline & 1000 & $86.7 \pm 9.7$ & $108.2 \pm 9.7$ & $92.9 \pm 8.3$ \\
\hline \multirow[t]{3}{*}{ TCEP } & 0 & nd & nd & nd \\
\hline & 500 & $113.2 \pm 10.4$ & $100.3 \pm 10.4$ & $99.9 \pm 5.6$ \\
\hline & 5000 & $94.2 \pm 8.2$ & $106.8 \pm 7.8$ & $108.6 \pm 4.2$ \\
\hline \multirow[t]{3}{*}{ TCPP } & 0 & nd & $150.4 \pm 6.5$ & nd \\
\hline & 500 & $101.8 \pm 3.6$ & $97.6 \pm 9.5$ & $101.7 \pm 3.3$ \\
\hline & 5000 & $106.2 \pm 5.9$ & $108.8 \pm 4.5$ & $83.2 \pm 8.6$ \\
\hline \multirow[t]{3}{*}{ TDCP } & 0 & nd & nd & nd \\
\hline & 500 & $101.0 \pm 9.4$ & $95.0 \pm 6.2$ & $83.2 \pm 9.7$ \\
\hline & 5000 & $112.1 \pm 9.4$ & $94.5 \pm 9.7$ & $98.4 \pm 8.6$ \\
\hline \multirow[t]{3}{*}{ TPhP } & 0 & nd & $162.1 \pm 8.8$ & nd \\
\hline & 100 & $99.7 \pm 7.7$ & $98.2 \pm 6.0$ & $95.6 \pm 3.4$ \\
\hline & 1000 & $85.4 \pm 8.9$ & $111.6 \pm 8.8$ & $94.7 \pm 8.7$ \\
\hline \multirow[t]{3}{*}{ EHDPP } & 0 & nd & nd & nd \\
\hline & 100 & $113.9 \pm 3.2$ & $108.5 \pm 7.3$ & $85.0 \pm 10.1$ \\
\hline & 1000 & $86.8 \pm 7.6$ & $89.4 \pm 3.7$ & $98.1 \pm 9.4$ \\
\hline \multirow[t]{3}{*}{ TEHP } & 0 & $205.4 \pm 6.9$ & $185.3 \pm 9.2$ & nd \\
\hline & 100 & $109.8 \pm 4.9$ & $96.8 \pm 6.5$ & $90.6 \pm 5.5$ \\
\hline & 1000 & $105.0 \pm 8.8$ & $89.4 \pm 7.7$ & $99.0 \pm 4.3$ \\
\hline \multirow[t]{3}{*}{ TCrP } & 0 & nd & nd & nd \\
\hline & 500 & $96.8 \pm 9.3$ & $94.1 \pm 8.1$ & $100.9 \pm 7.8$ \\
\hline & 5000 & $84.7 \pm 7.9$ & $110.2 \pm 3.1$ & $86.5 \pm 6.8$ \\
\hline
\end{tabular}

good recoveries and RSDs of TEHP also showed that the $\mathrm{GO} / \beta$ CD sol-gel coating fiber may be more suitable for extracting OPFRs than PDMS/DVB fiber.

\section{Conclusions}

In this work, a simple, low-cost and sensitive HS-SPME-GC/NPD method using $\mathrm{GO} / \beta$-CD sol-gel stainless steel coating fiber was proposed for the determination of nine OPFRs from water samples. Under the optimal conditions, the proposed method exhibits good precision, detection limit and linear response over a wide concentration range of OPFRs. The proposed method had been successfully applied for the determination of OPFRs in the real water samples with good recoveries and RSDs, including TEHP. Based on these highly efficient results, the proposed approach provides another new and alternative GObased SPME fiber for detecting OPFRs in water samples, which goes forward a step on our previous study. ${ }^{36}$ Moreover, this is also the first report about the preparation and application of GO-based composite sol-gel fiber. The novel coating showed higher extraction capability for most of OPFRs than the commercial fibers and GO sol-gel fiber. It also has the advantages of high stability, long lifetime, easy preparation and low cost. Both the single fiber repeatability and fiber-to-fiber reproducibility were satisfactory. Based on these features, the proposed fiber has a great potential for widespread use as an effective and useful extraction tool.

\section{Conflicts of interest}

There are no conflicts to declare.

\section{Acknowledgements}

This work was supported by the National Natural Science Foundation of China (Grants 21677058), the Fundamental Research Funds for the Central Universities (Grants 20205170506) and China Scholarship Fund (201706775016).

\section{References}

$1 \mathrm{P}$. Darnerud, Toxic effects of brominated flame retardants in man and in wild life, Environ. Int., 2003, 29, 841-853.

2 Stockholm Conference of Parties, Report of the Conference of the Parties of the Stockholm Convention on Persistent Organic Pollutants on the Work of Its Fourth Meeting: Stockholm Convention on Persistent Organic Pollutants, Geneva, 2009.

3 European Flame Retardants Association, Market Statistics, http://www.flameretardants.eu/DocShareNoFrame/docs/1/ HGJJCNFBPCEOKPEPPIPEICDF53V443-HA4YW3PDB348BT/ EFRA/docs/DLS/EFRA_web_11-2007_Market_statistics-1. pdf2007.

4 L. V. Dishaw, C. M. Powes, I. T. Ryde, S. C. Roberts, F. J. Seidler, T. A. Slotkin and H. M. Stapleton, Is the PentaBDE replacement, tris (1,3-dichloro-2-propyl) phosphate (TDCPP), a developmental neurotoxicant? Studies in PC12 cells, Toxicol. Appl. Pharmacol., 2011, 256, 281-289.

5 N. Van den Eede, A. C. Dirtu, H. Neels and A. Covaci, Analytical developments and preliminary assessment of 
human exposure to organophosphate flame retardants from indoor dust, Environ. Int., 2011, 37, 454-461.

6 X. Liu, K. Ji and K. Choi, Endocrine disruption potentials of organophosphate flame retardants and related mechanisms in H295R and MVLN cell lines and in zebrafish, Aquat. Toxicol., 2012, 114, 173-181.

7 J. D. Meeker, E. M. Copper, H. M. Stapleton and R. Hauser, Urinary metabolites of organophosphate flame retardants: temporal variability and correlations with house dust concentrations, Environ. Health Perspect., 2013, 121, 580-585.

8 T. Reemtsma, J. B. Quintana, R. Rodil, M. G. Lopez and I. Rodriguez, Organophosphorus flame retardants and plasticizers in water and air I. Occurrence and fate, Trends Anal. Chem., 2008, 27, 727-737.

9 I. Rodríguez, F. Calvo, J. B. Quintana, E. Rubí, R. Rodil and R. Cela, Suitability of solid-phase microextraction for the determination of organophosphate flame retardants and plasticizers in water samples, J. Chromatogr. A, 2006, 1108, 158.

10 Z. Q. Gao, Y. H. Deng, X. B. Hu, S. G. Yang, C. Sun and H. He, Determination of organophosphate esters in water samples using an ionic liquid-based sol-gel fiber for headspace solid-phase microextraction coupled to gas chromatography-flame photometric detector, $J$. Chromatogr. A, 2013, 1300, 141-150.

$11 \mathrm{~J}$. A. Andresen, A. Grundmann and K. Bester, Organophosphorus flame retardants and plasticisers in surface waters, Sci. Total Environ., 2004, 332, 155-166.

12 R. Rodil, J. B. Quintana and T. Reemtsma, Liquid chromatography-tandem mass spectrometry determination of nonionic organophosphorus flame retardants and plasticizers in wastewater Samples, Anal. Chem., 2005, 77, 3083-3089.

13 J. B. Quintana and T. Reemtsma, Potential of membraneassisted solvent extraction for the determination of phosphoric acid triesters in wastewater samples by liquid chromatography-tandem mass spectrometry, J. Chromatogr. A, 2006, 1124, 22-28.

14 M. García-López, I. Rodríguez and R. Cela, Development of a dispersive liquid-liquid microextraction method for organophosphorus flame retardants and plastizicers determination in water samples, J. Chromatogr. A, 2007, 1166, 9-15.

15 M. García-López, I. Rodríguez and R. Cela, Evaluation of liquid-liquid microextraction using polypropylene microporous membranes for the determination of organophosphorus flame retardants and plasticizers in water samples, Anal. Chim. Acta, 2008, 625, 145-153.

16 X. W. Wang, Y. Q. He, L. Lin, F. Zeng and T. G. Luan, Application of fully automatic hollow fiber liquid phase microextraction to assess the distribution of organophosphate esters in the Pearl River Estuaries, Sci. Total Environ., 2014, 470-471, 263-269.

17 C. L. Arthur and J. Pawliszyn, Solid phase microextraction with thermal desorption using fused silica optical fibers, Anal. Chem., 1990, 62, 2145-2148.
18 Z. Zhang, L. Zhu, Y. Ma, Y. Huang and G. Li, Preparation of polypyrrole composite solid-phase microextraction fiber coatings by sol-gel technique for the trace analysis of polar biological volatile organic compounds, Analyst, 2013, 138, 1156-1166.

19 Y. Li, Y. Wang, J. Zhang and C. Sun, Determination of 16 polycyclic aromatic hydrocarbons in water using fluorinated polyaniline-based solid-phase microextraction coupled with gas chromatography, Environ. Monit. Assess., 2012, 184, 4345-4353.

20 J. Lopez-Darias, J. L. Anderson, V. Pino and A. M. Afonso, Developing qualitative extraction profiles of coffee aromas utilizing polymeric ionic liquid sorbent coatings in headspace solid-phase microextraction gas chromatography-mass spectrometry, Anal. Bioanal. Chem., 2011, 401, 2965-2976.

21 D. Djozan, Y. Assadi and S. H. Haddadi, Anodized aluminum wire as a solid-phase microextraction fiber, Anal. Chem., 2001, 73, 4054-4058.

22 N. Chang, Z. Gu, H. Wang and X. Yan, Metal-organicframework-based tandem molecular sieves as a dual platform for selective microextraction and high-resolution gas chromatographic separation of n-alkanes in complex matrixes, Anal. Chem., 2011, 83, 7094-7101.

23 R. F. Jiang, F. Zhu, T. G. Luan, Y. X. Tong, H. Liu, G. F Ouyang and J. Pawliszyn, Carbon nanotube-coated solid-phase microextraction metal fiber based on sol-gel technique, $J$. Chromatogr. A, 2009, 1216, 4641-4647.

24 M. Sun, J. J. Feng, Y. N. Bu, X. J. Wang, H. M. Duan and C. N. Luo, Graphene coating bonded onto stainless steel wire as a solid-phase microextraction fiber, Talanta, 2015, 134, 200-205.

25 S. Y. Li, C. W. Lu, F. Zhu, R. F. Jiang and G. F. Ouyang, Preparation of $\mathrm{C}_{18}$ composite solid-phase microextraction fiber and its application to the determination of organochlorine pesticides in water samples, Anal. Chim. Acta, 2015, 873, 57-62.

26 Y. Y. Wen, Z. L. Niu, Y. L. Ma, J. P. Ma and L. X. Chen, Graphene oxide-based microspheres for the dispersive solid-phase extraction of non-steroidal estrogens from water samples, J. Chromatogr. A, 2014, 1368, 18-25.

27 S. H. Zhang, Z. Li, C. Wang and Z. Wang, Cyclodextrinfunctionalized reduced graphene oxide as a fiber coating material for the solid-phase microextraction of some volatile aromatic compounds, J. Sep. Sci., 2015, 38, 17111720.

28 Y. Guo, S. Guo, J. Ren, Y. Zhai, S. Dong and E. Wang, Cyclodextrin functionalized graphene nanosheets with high supramolecular recognition capability: synthesis and host_guest inclusion for enhanced electrochemical performance, ACS Nano, 2010, 4, 4001-4010.

29 C. Xu, J. Wang, L. Wan, J. Lin and X. Wang, Microwaveassisted covalent modification of graphene nanosheets with hydroxypropyl- $\beta$-cyclodextrin and its electrochemical detection of phenolic organic pollutants, J. Mater. Chem., 2011, 21, 10463-10471. 
30 G. Crini, Kinetic and equilibrium studies on the removal of cationic dyes from aqueous solution by adsorption onto a cyclodextrin polymer, Dyes Pigm., 2008, 77, 415-426.

31 J. Zhang, X. Shen and Q. Chen, Separation processes in the presence of cyclodextrins using molecular imprinting technology and ionic liquid cooperating approach, Curr. Org. Chem., 2011, 15, 74-85.

32 Y. P. Chin, S. Mohamad and M. R. B. Abas, Removal of parabens from aqueous solution using $\beta$-cyclodextrin cross-linked polymer, Int. J. Mol. Sci., 2010, 11, 3459-3471.

33 M. Raoov, S. Mohamad, M. R. Abas and H. Surikumaran, New macroporous $\beta$-cyclodextrin functionalized ionic liquid polymer as an adsorbent for solid phase extraction with phenols, Talanta, 2014, 130, 155-163.

34 C. López-de-Dicastillo, M. Gallur, R. Catalá, R. Gavara and P. Hernandez-Muñoz, Immobilization of $\beta$-cyclodextrin in ethylene-vinyl alcohol copolymer for active food packaging applications, J. Membr. Sci., 2010, 353, 184-191.

35 L. Qian, Y. Guan and H. Xiao, Preparation and characterization of inclusion complexes of a cationic $\beta$ cyclodextrin polymer with butylparaben or triclosan, Int. J. Pharm., 2008, 357, 244-251.
36 T. Sun, J. Jia, N. Fang and Y. Wang, Application of novel activated carbon fiber solid-phase microextraction to the analysis of chlorinated hydrocarbons in water by gas chromatography-mass spectrometry, Anal. Chim. Acta, 2005, 530, 33-40.

37 Y. Liu, Y. F. Shen and M. L. Lee, Porous layer solid phase microextraction using silica bonded phases, Anal. Chem., 1997, 69, 190-195.

38 J. X. Wang, D. Q. Jiang, Z. Y. Gu and X. P. Yan, Multiwalled carbon nanotubes coated fibers for solid-phase microextraction of polybrominated diphenyl ethers in water and milk samples before gas chromatography with electron-capture detection, J. Chromatogr. A, 2006, 1137, 814.

39 A. Medinia, M. F. Mousavi and M. Shamsipur, Nanostructured lead dioxide as a novel stationary phase for solid-phase microextraction, J. Chromatogr. A, 2006, 1134, 24-31.

40 J. X. Yu, L. Dong, C. Wu, L. Wu and J. Xing, Hydroxyfullerene as a novel coating for solid-phase microextraction fiber with sol-gel technology, J. Chromatogr. A, 2002, 978, 37-48. 